\title{
Review
}

\section{The biology of cytotoxic cell granule exocytosis pathway: granzymes have evolved to induce cell death and inflammation}

\author{
Julián Pardo a,b,*,1, Juan Ignacio Aguilo a , Alberto Anel ${ }^{\text {a }}$, Praxedis Martin ${ }^{\mathrm{c}}$, \\ Lars Joeckel $^{\mathrm{c}, \mathrm{d}}$, Christoph Borner ${ }^{\mathrm{d}}$, Reiner Wallich ${ }^{\mathrm{e}}$, Arno Müllbacher ${ }^{\mathrm{f}}$, \\ Christopher J. Froelich ${ }^{\mathrm{g}, * *, 1}$, Markus M. Simon ${ }^{\mathrm{c}, * * *, 1}$ \\ ${ }^{a}$ Dpto. Bioquímica y Biología Molecular y Celular, Facultad de Ciencias, Universidad de Zaragoza, Spain \\ ${ }^{\mathrm{b}}$ Fundación Aragón I+D/Gobierno de Aragón, Zaragoza, Spain \\ ${ }^{\mathrm{c}}$ Metschnikoff laboratory, Max-Planck Institute for Immunobiology, Freiburg, Germany \\ ${ }^{\mathrm{d}}$ Institute of Molecular Medicine and Cell Research, Center for Biochemistry and Molecular Research, Freiburg, Germany \\ ${ }^{\mathrm{e}}$ Institut für Immunologie, Universitätsklinikum Heidelberg, Heidelberg, Germany \\ ${ }^{\mathrm{f}}$ Viral Immunology, Division of Immunology and Genetics, John Curtin School of Medical Research, Australian National University, Canberra, Australia \\ ${ }^{\mathrm{g}}$ Department of Medicine, NorthShore University HealthSystem Research Institute, Evanston, IL, USA
}

Received 5 February 2009; accepted 13 February 2009

Available online 26 February 2009

\begin{abstract}
The granule exocytosis pathway of cytotoxic lymphocytes (Tc and NK cells) is critical for control of tumor development and viral infections. Granule-associated perforin and granzymes are key components in Tc cell-mediated function(s). On the basis of studies that showed granzymes $\mathrm{A}, \mathrm{B}, \mathrm{C}, \mathrm{K}$ and $\mathrm{M}$, to induce apoptosis in vitro, all granzymes were thought to also induce cell death in vivo. This review summarizes our present understanding of the biological processes elicited by purified granzyme A and granzyme as well as the processes induced by the more physiologically relevant cytotoxic cells secreting these proteases. The combined evidence supports the concept that the granule secretion pathway is not mono-specific but rather poly-functional including induction of pro-inflammatory cytokines, besides their widely appreciated apoptotic properties.
\end{abstract}

(c) 2009 Elsevier Masson SAS. All rights reserved.

Keywords: Granule exocytosis; Perforin; Granzymes; Cell death; Inflammation

\section{Introduction}

Cytotoxic T lymphocytes (Tc cells) and Natural Killer (NK) cells are key components of the host immune system that control infection by intracellular pathogens, primarily viruses and

\footnotetext{
* Corresponding author. Dpto. Bioquímica y Biología Molecular y Celular, Facultad de Ciencias, Universidad de Zaragoza, Pedro Cerbuna 12, 50009 Zaragoza, Spain. Tel.: +34 976762301; fax: +34 976762123 .

** Corresponding author. Evanston Northwestern Healthcare Research Institute, 1001 University Pl., Evanston, IL 60201. Tel.: +847 570 7660; fax: +847 5708025.

*** Corresponding author. Metschnikoff Laboratory, Max-Planck-Institut für Immunbiologie, Stübeweg 51, D-79108 Freiburg, Germany. Tel.: +497615108533; fax: +497615108529 .

${ }^{1}$ These authors share senior authorship.
}

neoplasms. Both cytotoxic effector cells act through either the death receptor pathway (i.e. Fas/FasL) and/or the granule secretory pathway. The latter is a phenomenon unique to mammalian vertebrate biology where biologically active molecules are secreted by the effector cells to penetrate the cytosol of the responding target cell. After engaging a target cell, cytoplasmic granules of the cytotoxic cell migrate toward the contact site (i.e., immunological synapse) and fuse with the plasma membrane. The released granule contents then diffuse toward the target cell [1], where perforin (PFN) facilitates intracellular delivery of granzymes (gzms), encoded in $10 \mathrm{gzm}$ genes in mice $(\mathrm{gzmA}$, $g z m B-G, g z m K, M$ and $N)$ and five genes in humans ( $g z m A$, $g z m B, g z m K, g z m H$ and $g z m M$; see Table 1) [2].

Although the concerted action of PFN with gzmA and gzmB is critical in the control of viral infections [2-5] their 
Table 1

In vivo granzyme expression found in several cell types.

\begin{tabular}{|c|c|c|c|}
\hline & Specie & $\begin{array}{l}\text { Enzyme-like } \\
\text { activity }\end{array}$ & In vivo expression ${ }^{\mathrm{a}}$ \\
\hline Granzyme A & Human, mouse & Tryptase & Tc cell, NK, NKT, $\gamma \delta$ \\
\hline Granzyme B & Human, mouse & ASPase & $\begin{array}{l}\text { Tc cell, NK, NKT, } \gamma \delta, \text { MC, } \\
\text { CD4, Bas, Mac, DC, Ser }\end{array}$ \\
\hline Granzyme C & Mouse & ? & $?$ \\
\hline Granzyme D & Mouse & Chymase & $?$ \\
\hline Granzyme E & Mouse & Chymase & $?$ \\
\hline Granzyme F & Mouse & Chymase & $?$ \\
\hline Granzyme $\mathrm{G}$ & Mouse & Chymase & $?$ \\
\hline Granzyme $\mathrm{H}$ & Human & Chymase & NK \\
\hline Granzyme K & $\begin{array}{l}\text { Human, } \\
\text { mouse }\end{array}$ & Tryptase & $\mathrm{NK}^{*}, \mathrm{NKT}, \mathrm{Tc}$ cell, $\gamma \delta$ \\
\hline Granzyme M & $\begin{array}{l}\text { Human, } \\
\text { mouse }\end{array}$ & METase & NK, NKT, $\gamma \delta$ \\
\hline Granzyme N & Mouse & $?$ & Sper \\
\hline
\end{tabular}

${ }^{\mathrm{a}}$ Cell type in which the protein has been found in vivo: Tc cell: cytotoxic $\mathrm{T}$ lymphocyte. NK: Natural Killer cell. NK*: CD56 (bright) NK cell. NKT: Natural Killer T cell. MC: mast cell. CD4: T helper cell. Bas: basophil. Mac: macrophage. $\gamma \delta: \gamma \delta \mathrm{T}$ cell. DC: dendritic cell. Ser: sertoli cells. Sper: spermatocytes. Mem CD8: memory $\mathrm{CD} 8^{+} \mathrm{T}$ cell.

importance in the elimination of tumor cells is less clear [6-9]. Our understanding of the biological roles played by other gzms, termed orphan gzms $(\mathrm{gzmC}-\mathrm{G}, \mathrm{K}, \mathrm{L}, \mathrm{N}$ and M) is even more rudimentary. What then is the totality of the biological function of the granule secretion pathway and why have so many varied specificities of gzms evolved to become participants $[10,11]$ ?

\section{Which granzymes are the real cytotoxic executioners?}

The original finding that gzms are contained within cytoplasmic granules of Tc or NK cells and are released upon ligation of the Tc receptor led to the concept that gzms may act extracellularly and intracellularly, thereby facilitating diverse processes, such as extracellular matrix degeneration, extravasation, inflammation and cytolysis [12]. However, the important discovery that, in the presence of PRF, gzms, initially gzmA [13] and later on also gzmB [14], induce apoptosis in target cells, focused research on their biological role in cell death and lead to the prediction that all gzms participate in the cytolytic processes mediated by NK/Tc cells. Support for this view has been underpinned by numerous in vitro studies [15] in which either purified mouse (Mo) or human $(\mathrm{Hu})$ gzms $(\mathrm{A}, \mathrm{B}, \mathrm{C}, \mathrm{H}, \mathrm{K}$ and $\mathrm{M})$ were used in combination with PRF from different species (human, mouse, rat) or other delivery agents including adenoviral particles, streptolysin O (SLO) and occasionally listeriolysin [16].

However, the apparent cytotoxicity observed for any purified gzm under in vitro conditions should be interpreted with caution since any serine protease is potentially toxic after intracellular delivery [17]. Many variables may influence the outcome of experiments designed to understand the function of individual gzms and are subject to a variety of interpretations. For example, conditions need to be clearly established to mirror physiological conditions and that gzms are effectively yet safely delivered into the targets before death parameters and other biochemical measurements can be meaningfully interpreted [18]. Also of concern are results obtained by using synergizing components from different species in protein- or cell-based cytotoxicity assays. For example, gzm orthologs may have distinct substrate specificities [19-21], which could result in in vitro artifacts.

Any putative cytotoxic function identified for a given purified gzm using such experimental systems, needs to be verified at the whole cell level using effector cells designed to selectively secrete the individual protease of interest.

This goal is achieved, at least in part, by using ex vivoderived Tc or NK cells from wild type (wt) and KO mice, which express almost exclusively gzmA and/or gzmB. One possible contaminant being gzmK, which is expressed in both, in vivo-derived and in vitro-propagated $\mathrm{Tc}$ cells [22]. Depending on the in vitro conditions, propagated Tc cell lines may also express gzmC-G [7,22-25]. Studies with ex vivoderived Tc cells from gzm KO mice, clearly demonstrated that cell death delivery is markedly impaired in the absence of gzmB [26-28] and even more so when both gzmA and gzmB are absent [27-29]. The observation that all ex vivo Tc cells similarly express gzmK, but in the absence of gzmB cytolytic activity is greatly diminished, indicates that gzmB is the main cytotoxic effector molecule whereas gzmA and gzmK are less cytotoxic, or not at all.

Finally, when using in vitro cytolytic assays it is very important that multiple parameters such as membrane damage, PS externalization, mitochondrial depolarization, ROS induction and DNA breakage are analyzed by flow cytometry. In addition the proliferation potential of affected target cells (clonogenic assay, thymidine incorporation) needs to be evaluated. Only after cytotoxic capacity of a gzm using isolated protease assays are assessed with relevant cytotoxic cell systems like ex vivo-derived virus-immune Tc cells and results are compatible can one assign biological function to individual gzms.

\section{Cell death induced by purified gzmA and by Tc cells containing $\operatorname{gzmA}\left(\operatorname{gzmA}^{+} \mathbf{T c}\right)$}

The first report that native mouse gzmA (nMogzmA) is a cytotoxic serine protease was published by the Henkart lab showing that treatment of EL-4 target cells with isolated nMogzmA and native mouse PFN (nMoPFN) caused significant target DNA breakdown [13]. Shortly thereafter, Greenberg and Shi isolated native rat gzmA (nRagzmA) observing that the protease could induce apoptosis (DNA fragmentation) in the presence of native rat PFN (nRaPRF) [14]. At the same time, Shiver et al. reported that transfection of rat basophilic leukemia (RBL) cells with both MogzmA and MoPFN genes endowed the line with the potential to induce target cell DNA breakdown [30]. Studies of the mechanism by which gzmA kills cells remained unknown and awaited the in vitro expression and isolation of gzmA.

Lieberman's group, using recombinant HugzmA (rHugzmA) and nRaPFN, described biochemical schemes for 
gzmA-mediated cytotoxicity (Fig. 1) [15]. After release into the cytosol of target cells, gzmA enters the mitochondrial matrix as well as the nucleus. Within mitochondria, gzmA processes a complex I mitochondrial protein, NDUFS3, stimulating the generation of reactive oxygen species (ROS). The increased level of ROS then activates cellular detoxification mechanisms including the ER-associated SET complex [15]. The activated SET complex translocates to the nucleus facilitating the repair of the ROS-induced oxidative DNA damage. However, gzmA cleaves several components of the SET complex, leading to the release of the endonuclease, NM23H1, which induces single-stranded DNA damage (nicking), resulting in cell death.

Recent studies however have cast some doubt as to whether isolated $\mathrm{Hu}$ - or MogzmA is able to induce cell death under normal physiological conditions. Indications are that when $\mathrm{Hu}-$ and MogzmA are used at concentrations previously reported to be cytotoxic (micromolar), the protease induces, in concert with HuPFN or SLO rapid membranolysis [31]. However, if either HugzmA or MogzmA is used under conditions employed to demonstrate the cytolytic potential of gzmB (nanomolar concentrations), cell death was not observed. This was established during multiple short term readouts as well as in long term proliferation assays [31]. Thus, the described biochemical effects attributed to gzmA may have occurred in necrotic cells, which became permeable to the gzm.

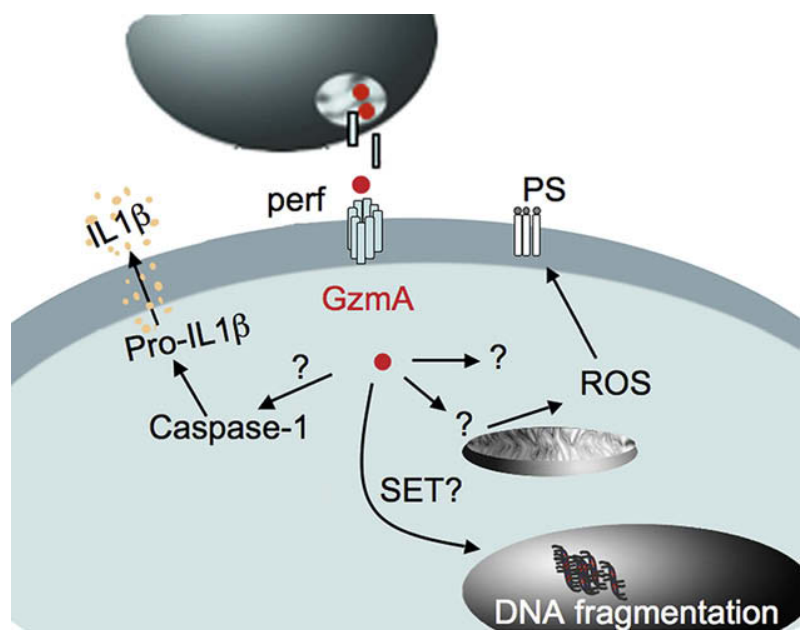

Fig. 1. The role and mechanism of ex vivo Tc cells delivered gzmA on target cells. Tc cells recognize and bind to target cells (virus infected or transformed cells) and secrete perforin (perf) and granzymes (gzm). Perf facilitates gzm entry into the cytosol of the target cell where it can induce several processes. GzmA can induce two different processes. Left: in macrophages GzmA is able to promote cleavage of Pro-IL1 $\beta$ to generate mature IL1 $\beta$, which is secreted in the extracellular media. This process depends on caspase-1 activity. By this mean GzmA is able to activate pro-inflammatory signals. In fact absence of GzmA improves mouse survival after LPS challenge. Right: GzmA may induce cell death in some but not all target cells. However, this process may depend on additional molecules present in the Tc cell or the target cell. The mechanism activated by GzmA in susceptible target cells is not clear at present. ROS contribute to some extent to PS translocation, but the role of the SET complex (Ape-1, SET and the endonuclease NM23H1) is still pending of validation by using Tc cells.
Since $\mathrm{Hu} / \operatorname{MogzmA}$ is generally non-toxic in vitro, except under extreme conditions, the protease is unlikely to induce cell death at concentrations mirroring those after secretion by a cytotoxic effector cell. Thus data derived from experiments that examine the cytotoxicity of ex vivo immune Tc and $\mathrm{NK}$ cells secreting the gzm will be more informative. Analysis of data derived from experiments using ex vivo mouse $\mathrm{gzmA}^{+} \mathrm{Tc}$ cells revealed their potential to induce many of the characteristic signs of cell death, however only in some (EL-4 thymoma or A1.1 and TA3 lymphoma cells) but not other (mouse embryo fibroblasts (MEF), L1210 leukemia cells) target cells, a phenomenon at present not understood [27,28,32] (and J. Pardo and M.M. Simon, unpublished observations).

Importantly however, compared to ex vivo mouse $\mathrm{gzmB}^{+}$ and wt Tc cells, the potency of cytotoxicity is low [32] and contrasts with data obtained using purified MogzmA [33]. Furthermore, when ex vivo virus-immune Tc cells were used $[27,28,32]$ the pattern of DNA fragmentation observed was oligonucleosomal and not single-stranded DNA nicking, as shown for HugzmA [33]. In addition, cleavage of putative gzmA substrates such as Ape-1, HMGB2, Ku70, histones, and NDUFS3 is not apparent under conditions where gzmAsecreting Tc cells are employed [34-39].

Since purified MogzmA is not cytotoxic, mouse gzmA $^{+} \mathrm{Tc}$-induced killing of specific target cells must depend on either additional molecules present in cytoplasmic granules of the cytotoxic cells (e.g., other gzms and/or cathepsins), or a different quality of Tc-delivered gzmA or on an unusual susceptibility of the target cell to the effector gzmA $^{+}$Tc cells [28]. The inconsistencies observed between the cytotoxic activity of purified MogzmA and those mediated via gzmA ${ }^{+} \mathrm{Tc}$ necessitate re-evaluation of published data with experimental designs in which expression of all gzms beside gzmA is inhibited by siRNA or by gene inactivation.

\section{Cell death induced by purified gzmB or by selectively gzmB expressing $\mathrm{Tc}$ cells $\left(\mathrm{gzmB}^{+} \mathrm{Tc}\right)$}

The observation that mouse, gzmB deficient, Tc cells failed to induce rapid DNA fragmentation was the first evidence indicating that gzmB is crucial for cell-mediated induction of apoptosis [26]. Since then, studies on the molecular mechanisms of gzmB-mediated apoptosis have employed native and more recently recombinant proteins delivered by PFN derived from various species (human, mouse, rat) or other delivery systems [18,40-42]. However, as mentioned above, the indiscriminate employment of gzmB, PFN and target cells' sources from different species does complicate data analysis.

The present consensus view on Hu/MogzmB-initiated apoptosis is a two pathway mechanism (Fig. 2): gzmB either directly activates the executioner caspases ( -3 and -7$)$ [43-45]; or caspase activation is initiated indirectly through mitochondrial permeabilization promoted by gzmB-mediated cleavage of Bid [46-50]. In the latter case, activated tBid induces oligomerization of $\mathrm{Bak} / \mathrm{Bax}$ at the outer mitochondrial membrane leading to cytochrome c release and the assembly of the apoptosome, with subsequent activation of caspase 9 followed by 


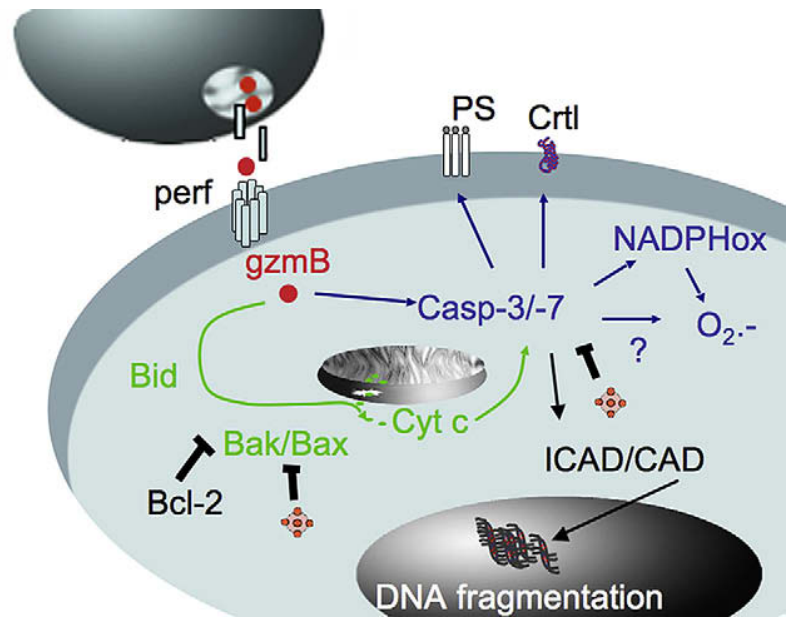

Fig. 2. The role and mechanism of $e x$ vivo Tc cells delivered-gzmB on target cells. GzmB induces apoptotic cell death by two different pathways. Direct caspase-3 or caspase-7 activation is critical to induce PS and calreticulin (Crtl) translocation, ROS production and contributes to mitochondrial depolarization. Bid cleavage generates the active $\mathrm{tBid}$ form that induces Bak and/or Bax activation and promotes cytochrome c (cyt c) release from the mitochondria and subsequently caspase activation (by apoptosome formation). In addition, gzmB is able to induce ROS production outside of the mitochondria by a caspase dependent activation of NADPH oxidase(s) (Aguiló et al., unpublished observations). By blocking simultaneously caspase-3/-7 and $\mathrm{Bak} / \mathrm{Bax}$ all apoptotic features are inhibited but gzmB is still able to kill the cells by an unknown mechanism. The pleiotropy of gzmB-induced cell death is likely due to viral pressure during evolution, since viruses are able to interfere at different levels of the apoptotic machinery.

caspases 3 and 7. Interestingly, purified HugzmB and MogzmB were reported to have a strikingly different substrate preference for Bid and caspase-3 respectively [19-21]. Therefore in mice gzmB is suggested to initiate death mainly through caspase-3 (s.a. below for gzmB ${ }^{+} \mathrm{Tc}$ cell) while HugzmB gains the capacity to cleave Bid if counter-regulatory mechanisms dampen direct caspase- 3 activation.

To evaluate if the death pathway(s) induced by purified gzms are the same or similar to those induced by ex vivoderived mouse $\mathrm{gzmB}^{+} \mathrm{Tc}$ cells (i.e., $\mathrm{gzmA}^{-/-} \mathrm{Tc}$ cells) experiments were performed using target cells (MEFs) with defects in either the effector caspases (MEF.casp3 $\times 7^{-1-}$ ) or mitochondrial proteins (MEF.Bid-/-, MEF.Bak/Bax-/-) and cell death was monitored by flow based annexin reactivity, mitochondrial depolarization, ROS generation and proliferation potential [22]. $\mathrm{GzmB}^{+} \mathrm{Tc}$ cells triggered $\mathrm{Bid} / \mathrm{Bak} / \mathrm{Bax}$ deficient targets to undergo cell death, associated with caspase-3/-7 dependent PS translocation, $\Delta \Psi_{\mathrm{m}}$ loss and ROS production. On the other hand, in targets lacking caspase- 3 and caspase- 7, gzmB $^{+} \mathrm{Tc}$-mediated cell death was accompanied by processing of $\mathrm{Bid}$, leading to $\Delta \Psi_{\mathrm{m}}$ loss and cytochrome $\mathrm{c}$ release, consistent with results obtained with HugzmB [51]. Blockade of both death pathways prevented PS translocation mitochondrial depolarization and ROS production, but not cell death [22].

The finding that mouse ex vivo LCMV-immune [22,45] as well as in vitro-propagated alloreactive Tc $[22,45]$ are able to induce, via gzmB, both pathways suggests that the differential proteolytic activity observed with purified MogzmB [19-21] does not reflect its actual potential, when delivered by intact $\mathrm{Tc} / \mathrm{NK}$ cells. The fact that neither inhibition of Bcl-2 nor of caspases alone prevented cell death elicited by human NK cell-derived gzmB [52], extend this interpretation to the human system and suggests that both, human and mouse $\mathrm{Tc} /$ NK cells are able to independently activate both, the mitochondrial and the caspase death pathways.

Taking all the data into consideration $[22,28,32,52-54]$ the following scheme can be proposed (Fig. 2): Depending on the concentration of gzmB delivered intracellularly, the quantities of substrates and counter-regulatory processes which may suppress apoptosis (Smac/Diablo), gzmB does initiate cell death by engaging different facets of the death program: one depends on mitochondrial $\mathrm{Bid} / \mathrm{Bak} / \mathrm{Bax}$ regulated cytochrome c release followed by caspase activation and the other involves direct activation of the executioner caspases. Finally, if this bi-functional death pathway is completely blocked, gzmB retains the capacity to kill the target cell by a yet undefined additional pathway [22].

This plasticity of mouse and most probably also human gzmB $^{+} \mathrm{Tc}$ to induce target cell apoptosis is most likely due to evolutionary pressures exerted by pathogens attempting to evade immune elimination. One example being the orthopoxviruses, which encode serine protease inhibitors that severely curtail FasL/Fas-mediated cell death. Thus mice deficient in PFN and/or gzmA $\times \mathrm{B}$ are unable to control mouse pox, ectromelia (ECTV) [55]. Recent observations by us, that the ECTV is unable to inhibit apoptosis induced by $\mathrm{gzmB}^{+} \mathrm{Tc}$ in competent cells, support this interpretation (Pardo et al., unpublished results). However, ECTV does block gzmB ${ }^{+}$Tcmediated apoptosis in caspase $3 \times 7$ or Bak $\times$ Bax deficient cells pointing to the importance of having multiple proapoptotic pathways activated by gzmB to combat viral infections and poxviruses in particular.

\section{Cell death induced by gzms other than A and B}

Although purified gzmC, gzmK, gzmH and gzmM were reported to induce cell death when delivered by a variety of agents, such as streptolysin O, perforin or liposomes (ProJect) their contribution to $\mathrm{Tc} / \mathrm{NK}$ cell-mediated protective and/or inflammatory processes is not known (Fig. 3).

\subsection{GzmC}

Purified mouse gzmC was reported to induce cell death, accompanied by some (PS translocation, chromatin condensation and $\Delta \Psi_{\mathrm{m}}$ loss) but not other apoptotic features (oligonucleosomal DNA fragmentation, caspase activation, Bid cleavage or cytochrome c release) [56].

The expression of gzmC protein has not been demonstrated in vivo. However the finding that a) ex vivo Tc cells from intravenously but not intraperitoneally LCMV infected mice express gzmC transcripts [22,57], b) in vitro generated Tc cells from $\mathrm{CatC}-1-$ mice (mice deficient in the granzyme processing enzyme, dipeptidyl peptidase I) express gzmC after 


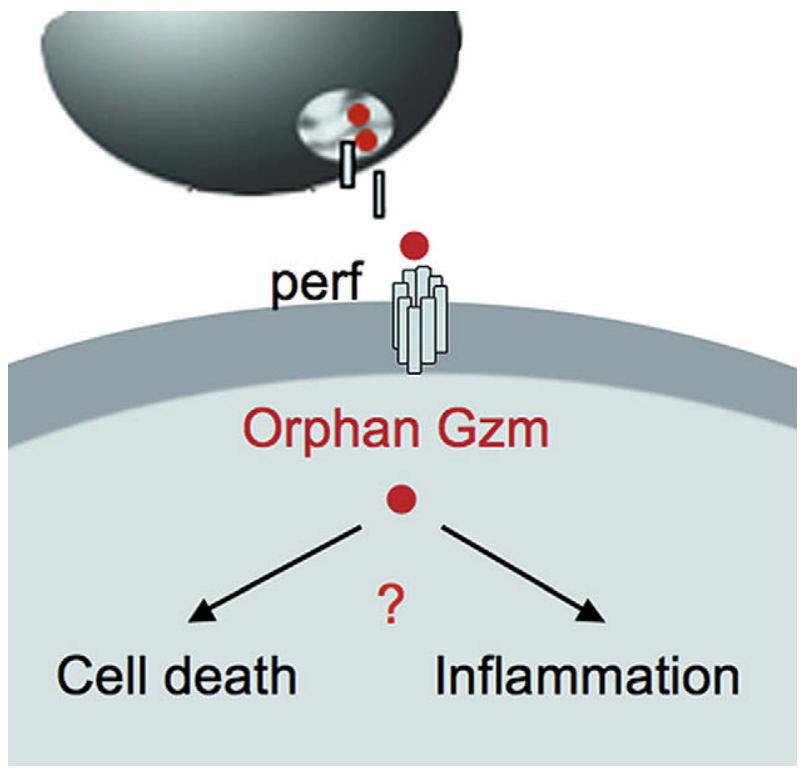

Fig. 3. The role and mechanism of ex vivo Tc cells delivered-orphan gzms on target cells. Nothing is known about the mechanism and consequences of ex vivo Tc cell-derived orphan gzms with in vitro cytotoxic potential (gzmC, gzmK, gzmH and gzmM). They may promote cell death, pro-inflammatory signals or other processes, but neither of them has been shown yet by using Tc or NK cells.

secondary activation [25], the blocking of which via RNAi partially rescued cell death elicited by these Tc cells, and c) mice deficient in gzmB, gzmC and gzmF are more resistant to tumor challenge than those deficient in gzmB alone [7], suggests that gzmC may contribute to $\mathrm{Tc} / \mathrm{NK}$ cell-mediated proapoptotic processes in vivo. However, since the protein has not been found in vivo, it still remains speculative if it plays a role during cell death induced by killer cells.

\subsection{GzmH}

Contradictory results have been reported for the proapoptotic potential of gzmH. Cell death induced by purified human gzmH was shown in one study to be caspase independent and accompanied by PS translocation, $\Delta \Psi_{\mathrm{m}}$ loss, ROS generation, DNA degradation, and chromatin condensation, but not Bid or ICAD cleavage nor cytochrome c release [58]. The other report showed Bid and ICAD cleavage, cytochrome c release and caspase dependency of apoptosis [59].

Although gzmH is strongly expressed by human NK cells, its involvement in cytotoxic processes is questionable. Independent studies have shown that inhibition of gzmB in gzmH positive human NK cells leads to nearly total abolition of their cytotoxic potential $[52,60,61]$.

\subsection{GzmK}

Contradictory reports also exist for the cytotoxic potential of HugzmK $[62,63]$. One study suggests that HugzmK induces processes, similar to HugzmA, including SET and Ape-1 cleavage and NM23H1 nuclear translocation but no caspase-3 activation [63]. The same group presented more recent data, indicating that gzmK induces Bid cleavage and cytochrome $\mathrm{c}$ release [62]. It is possible that caspase-3 was not activated even when cytochrome $\mathrm{c}$ was released from mitochondria due to the presence of inhibiting IAPs in the target cell $[64,65]$. However, caspase- 3 was readily activated by gzmB under similar conditions $[62,63]$.

Although gzmK is expressed by ex vivo LCMV-immune Tc cells, independent of their gzmA and/or gzmB expression $[22,66,67]$, its biological function remains elusive.

\subsection{GzmM}

Purified human gzmM was reported to induce cell death in Jurkat cells with ROS production, caspase activation, ICAD cleavage and DNA fragmentation $[68,69]$ or in the absence of any apoptotic feature [70,71]. Further more, purified human gzmM was found to cleave alpha-tubulin, thereby interfering with the integrity of the cytoskeleton [72]. As with gzmH, gzmM seems to be dispensable for NK cell induced cell death processes. However, the finding that gzmM deficient mice are slightly more susceptible to MCMV infection, but not ECTV infections, than wt mice suggests a possible role of this protease in the control of some viruses but not others [73].

\section{GzmA and $\operatorname{gzmA}^{+} \mathrm{Tc} / \mathrm{NK}$ cells induce pro- inflammatory cytokines}

As outlined above gzmA per se does not appear to induce cytotoxic effects after intracellular delivery into target cells thus leaving the question as to its biological function during an immune response open. Some clues as to that function has emerged recently from evidence which suggests that $\mathrm{Hu} /$ MogzmA may function as an inducer of pro-inflammatory cytokines [31]. Purified HugzmA as well as human NK cells selectively secreting gzmA induced human monocytes to secrete IL-1 and TNF in a caspase-1 dependent manner. Equally important, MogzmA and mouse $\operatorname{gzmA}^{+} \mathrm{Tc}$ cells induce IL1 $\beta$ release from primary mouse macrophages and gzmA KO mice were found to resist LPS-induced toxicity [31]. Thus the granule secretory pathway does appear to play an already previous suggested role in inflammation with gzmA acting as an endogenous modulator [12,74-76]

\section{Conclusions and future perspectives}

Overall data gathered from studies using ex vivo-derived Tc cells, rather than from experiments using purified protease preparations, indicate that apoptosis induced by the granule exocytosis pathway is critically dependent on PFN and gzmB. Although purified orphan gzms have been shown to induce cell death in vitro, the biological significance of these findings has still to be elucidated. Furthermore, it is now becoming clear that the granule secretion pathway is not uniquely focused on the elimination of target cells but instead, gzmA and perhaps some or all of the orphan gzms may contribute to 
host defense through the induction of Tc/NK-mediated proinflammatory cytokines. Further studies are required to elucidate the molecular basis and biological relevance of these phenomena. Finally, it is becoming more evident that other, non-cytotoxic functions, of gzms [83] have evolved in order to combat viruses [77,78] or regulate extracellular matrix dependent processes like atherosclerosis, anoikis or extravasation [79-82].

\section{Acknowledgments}

We would like to thank our many collaborators through the years. This work was supported in part by: Gobierno de Aragón (PI076/08), Ministerio de Ciencia e Innovación (SAF2008-02139), the Alexander von Humboldt Foundation and Fundación Agencia Aragonesa para Investigación y Desarrollo (ARAID) to J.P; SAF2004-03058 and SAF200765144 to A.A.; the Excellence Initiative of the German Federal and State Governments (EXC 294, bioss Cluster and GSC-4, Spemann Graduate School), the Deutsche Forschungsgesellschaft (GRK1104), the Jose Carreras Leukemia Foundation of Germany and the Bundesministerium für Bildung und Forschung (BMBF, HepatoSys) to C.B.; 5RO1AI04494-03 to C.J.F, NH\&MRC project grant S4221078 to A.M. We would like to apologize for not citing many additional relevant publications because of space limitations.

\section{References}

[1] G. Bossi, G.M. Griffiths, CTL secretory lysosomes: biogenesis and secretion of a harmful organelle, Semin. Immunol. 17 (2005) 87-94.

[2] J.H. Russell, T.J. Ley, Lymphocyte-mediated cytotoxicity, Annu. Rev. Immunol. 20 (2002) 323-370.

[3] A. Mullbacher, P. Waring, R. Tha Hla, T. Tran, S. Chin, T. Stehle, C. Museteanu, M.M. Simon, Granzymes are the essential downstream effector molecules for the control of primary virus infections by cytolytic leukocytes, Proc. Natl. Acad. Sci. U.S.A. 96 (1999) 13950-13955.

[4] R.A. Pereira, M.M. Simon, A. Simmons, Granzyme A, a noncytolytic component of $\mathrm{CD} 8(+)$ cell granules, restricts the spread of herpes simplex virus in the peripheral nervous systems of experimentally infected mice, J. Virol. 74 (2000) 1029-1032.

[5] L. Riera, M. Gariglio, G. Valente, A. Mullbacher, C. Museteanu, S. Landolfo, M.M. Simon, Murine cytomegalovirus replication in salivary glands is controlled by both perforin and granzymes during acute infection, Eur. J. Immunol. 30 (2000) 1350-1355.

[6] J. Pardo, S. Balkow, A. Anel, M.M. Simon, Granzymes are essential for natural killer cell-mediated and perf-facilitated tumor control, Eur. J. Immunol. 32 (2002) 2881-2887.

[7] P.A. Revell, W.J. Grossman, D.A. Thomas, X. Cao, R. Behl, J.A. Ratner, Z.H. Lu, T.J. Ley, Granzyme B and the downstream granzymes C and/or $\mathrm{F}$ are important for cytotoxic lymphocyte functions, J. Immunol. 174 (2005) 2124-2131.

[8] J.E. Davis, M.J. Smyth, J.A. Trapani, Granzyme A and B-deficient killer lymphocytes are defective in eliciting DNA fragmentation but retain potent in vivo anti-tumor capacity, Eur. J. Immunol. 31 (2001) 39-47.

[9] M.J. Smyth, S.E. Street, J.A. Trapani, Cutting edge: granzymes A and B are not essential for perforin-mediated tumor rejection, J. Immunol. 171 (2003) 515-518.

[10] P. Bolitho, I. Voskoboinik, J.A. Trapani, M.J. Smyth, Apoptosis induced by the lymphocyte effector molecule perforin, Curr. Opin. Immunol. 19 (2007) 339-347.
[11] S.M. Raja, S.S. Metkar, C.J. Froelich, Cytotoxic granule-mediated apoptosis: unraveling the complex mechanism, Curr. Opin. Immunol. 15 (2003) 528-532.

[12] M.D. Kramer, M.M. Simon, Are proteinases functional molecules of T lymphocytes? Immunol. Today 8 (1987) 140-142.

[13] M.P. Hayes, G.A. Berrebi, P.A. Henkart, Induction of target cell DNA release by the cytotoxic $\mathrm{T}$ lymphocyte granule protease granzyme A, J. Exp. Med. 170 (1989) 933-946.

[14] L. Shi, C.M. Kam, J.C. Powers, R. Aebersold, A.H. Greenberg, Purification of three cytotoxic lymphocyte granule serine proteases that induce apoptosis through distinct substrate and target cell interactions, J. Exp. Med. 176 (1992) 1521-1529.

[15] D. Chowdhury, J. Lieberman, Death by a thousand cuts: granzyme pathways of programmed cell death, Annu. Rev. Immunol. 26 (2008) $389-420$.

[16] C.J. Froelich, K. Orth, J. Turbov, P. Seth, R. Gottlieb, B. Babior, G.M. Shah, R.C. Bleackley, V.M. Dixit, W. Hanna, New paradigm for lymphocyte granule-mediated cytotoxicity. Target cells bind and internalize granzyme B, but an endosomolytic agent is necessary for cytosolic delivery and subsequent apoptosis, J. Biol. Chem. 271 (1996) 29073-29079.

[17] M.S. Williams, P.A. Henkart, Apoptotic cell death induced by intracellular proteolysis, J. Immunol. 153 (1994) 4247-4255.

[18] C.J. Froelich, S.S. Metkar, S.M. Raja, Granzyme B-mediated apoptosis the elephant and the blind men? Cell Death Differ. (2004).

[19] S.P. Cullen, C. Adrain, A.U. Luthi, P.J. Duriez, S.J. Martin, Human and murine granzyme B exhibit divergent substrate preferences, J. Cell Biol. 176 (2007) 435-444.

[20] D. Kaiserman, C.H. Bird, J. Sun, A. Matthews, K. Ung, J.C. Whisstock, P.E. Thompson, J.A. Trapani, P.I. Bird, The major human and mouse granzymes are structurally and functionally divergent, J. Cell Biol. 175 (2006) 619-630.

[21] L. Casciola-Rosen, M. Garcia-Calvo, H.G. Bull, J.W. Becker, T. Hines, N.A. Thornberry, A. Rosen, Mouse and human granzyme B have distinct tetrapeptide specificities and abilities to recruit the Bid pathway, J. Biol. Chem. 282 (2007) 4545-4552.

[22] J. Pardo, R. Wallich, P. Martin, C. Urban, A. Rongvaux, R.A. Flavell, A. Mullbacher, C. Borner, M.M. Simon, Granzyme B-induced cell death exerted by ex vivo CTL: discriminating requirements for cell death and some of its signs, Cell Death Differ. 15 (2008) 567-579.

[23] A. Kelso, E.O. Costelloe, B.J. Johnson, P. Groves, K. Buttigieg, D.R. Fitzpatrick, The genes for perforin, granzymes A-C and IFNgamma are differentially expressed in single $\mathrm{CD} 8(+) \mathrm{T}$ cells during primary activation, Int. Immunol. 14 (2002) 605-613.

[24] N.J. Waterhouse, V.R. Sutton, K.A. Sedelies, A. Ciccone, M. Jenkins, S.J. Turner, P.I. Bird, J.A. Trapani, Cytotoxic T lymphocyte-induced killing in the absence of granzymes A and B is unique and distinct from both apoptosis and perforin-dependent lysis, J. Cell Biol. 173 (2006) 133-144.

[25] Y. Getachew, H. Stout-Delgado, B.C. Miller, D.L. Thiele, Granzyme C supports efficient CTL-mediated killing late in primary alloimmune responses, J. Immunol. 181 (2008) 7810-7817.

[26] J.W. Heusel, R.L. Wesselschmidt, S. Shresta, J.H. Russell, T.J. Ley, Cytotoxic lymphocytes require granzyme B for the rapid induction of DNA fragmentation and apoptosis in allogeneic target cells, Cell 76 (1994) 977-987.

[27] M.M. Simon, M. Hausmann, T. Tran, K. Ebnet, J. Tschopp, R. ThaHla, A. Mullbacher, In vitro- and ex vivo-derived cytolytic leukocytes from granzyme $\mathrm{A} \times \mathrm{B}$ double knockout mice are defective in granule-mediated apoptosis but not lysis of target cells, J. Exp. Med. 186 (1997) 1781-1786.

[28] J. Pardo, S. Balkow, A. Anel, M.M. Simon, The differential contribution of granzyme A and granzyme B in cytotoxic T lymphocyte-mediated apoptosis is determined by the quality of target cells, Eur. J. Immunol. 32 (2002) 1980-1985.

[29] S. Shresta, T.A. Graubert, D.A. Thomas, S.Z. Raptis, T.J. Ley, Granzyme A initiates an alternative pathway for granule-mediated apoptosis, Immunity 10 (1999) 595-605. 
[30] J.W. Shiver, L. Su, P.A. Henkart, Cytotoxicity with target DNA breakdown by rat basophilic leukemia cells expressing both cytolysin and granzyme A, Cell 71 (1992) 315-322.

[31] S.S. Metkar, C. Menaa, J. Pardo, B. Wang, R. Wallich, M. Freudenberg, S. Kim, S.M. Raja, L. Shi, M.M. Simon, C.J. Froelich, Human and mouse granzyme A induce a proinflammatory cytokine response, Immunity (2008).

[32] J. Pardo, A. Bosque, R. Brehm, R. Wallich, J. Naval, A. Mullbacher, A. Anel, M.M. Simon, Apoptotic pathways are selectively activated by granzyme A and/or granzyme B in CTL-mediated target cell lysis, J. Cell Biol. 167 (2004) 457-468.

[33] P.J. Beresford, Z. Xia, A.H. Greenberg, J. Lieberman, Granzyme A loading induces rapid cytolysis and a novel form of DNA damage independently of caspase activation, Immunity 10 (1999) 585-594.

[34] Z. Fan, P.J. Beresford, D.Y. Oh, D. Zhang, J. Lieberman, Tumor suppressor NM23-H1 is a granzyme A-activated DNase during CTLmediated apoptosis, and the nucleosome assembly protein SET is its inhibitor, Cell 112 (2003) 659-672.

[35] D. Martinvalet, P. Zhu, J. Lieberman, Granzyme A induces caspaseindependent mitochondrial damage, a required first step for apoptosis, Immunity 22 (2005) 355-370.

[36] D. Martinvalet, D.M. Dykxhoorn, R. Ferrini, J. Lieberman, Granzyme A cleaves a mitochondrial complex I protein to initiate caspase-independent cell death, Cell 133 (2008) 681-692.

[37] P. Zhu, D. Zhang, D. Chowdhury, D. Martinvalet, D. Keefe, L. Shi, J. Lieberman, Granzyme A, which causes single-stranded DNA damage, targets the double-strand break repair protein Ku70, EMBO Rep. 7 (2006) 431-437.

[38] D. Zhang, M.S. Pasternack, P.J. Beresford, L. Wagner, A.H. Greenberg, J. Lieberman, Induction of rapid histone degradation by the cytotoxic $\mathrm{T}$ lymphocyte protease granzyme A, J. Biol. Chem. 276 (2001) 3683-3690.

[39] Z. Fan, P.J. Beresford, D. Zhang, J. Lieberman, HMG2 interacts with the nucleosome assembly protein SET and is a target of the cytotoxic Tlymphocyte protease granzyme A, Mol. Cell. Biol. 22 (2002) 2810-2820.

[40] J.A. Trapani, V.R. Sutton, Granzyme B: pro-apoptotic, antiviral and antitumor functions, Curr. Opin. Immunol. 15 (2003) 533-543.

[41] R.C. Bleackley, A molecular view of cytotoxic T lymphocyte induced killing, Biochem. Cell Biol. 83 (2005) 747-751.

[42] S.P. Cullen, S.J. Martin, Mechanisms of granule-dependent killing, Cell Death Differ. 15 (2008) 251-262.

[43] A.J. Darmon, D.W. Nicholson, R.C. Bleackley, Activation of the apoptotic protease CPP32 by cytotoxic T-cell-derived granzyme B, Nature 377 (1995) 446-448.

[44] S.S. Metkar, B. Wang, M.L. Ebbs, J.H. Kim, Y.J. Lee, S.M. Raja, C.J. Froelich, Granzyme B activates procaspase-3 which signals a mitochondrial amplification loop for maximal apoptosis, J. Cell Biol. 160 (2003) 875-885.

[45] D.A. Thomas, L. Scorrano, G.V. Putcha, S.J. Korsmeyer, T.J. Ley, Granzyme B can cause mitochondrial depolarization and cell death in the absence of BID, BAX, and BAK, Proc. Natl. Acad. Sci. U.S.A. 98 (2001) 14985-14990.

[46] J.B. Alimonti, L. Shi, P.K. Baijal, A.H. Greenberg, Granzyme B induces BID-mediated cytochrome c release and mitochondrial permeability transition, J. Biol. Chem. 276 (2001) 6974-6982.

[47] J.A. Heibein, I.S. Goping, M. Barry, M.J. Pinkoski, G.C. Shore, D.R. Green, R.C. Bleackley, Granzyme B-mediated cytochrome c release is regulated by the Bcl-2 family members Bid and Bax, J. Exp. Med. 192 (2000) 1391-1402.

[48] M.J. Pinkoski, N.J. Waterhouse, J.A. Heibein, B.B. Wolf, T. Kuwana, J.C. Goldstein, D.D. Newmeyer, R.C. Bleackley, D.R. Green, Granzyme B-mediated apoptosis proceeds predominantly through a Bcl-2-inhibitable mitochondrial pathway, J. Biol. Chem. 276 (2001) 12060-12067.

[49] V.R. Sutton, J.E. Davis, M. Cancilla, R.W. Johnstone, A.A. Ruefli, K. Sedelies, K.A. Browne, J.A. Trapani, Initiation of apoptosis by granzyme B requires direct cleavage of Bid, but not direct granzyme B-mediated caspase activation, J. Exp. Med. 192 (2000) 1403-1414.

[50] N.J. Waterhouse, K.A. Sedelies, K.A. Browne, M.E. Wowk, A. Newbold, V.R. Sutton, C.J. Clarke, J. Oliaro, R.K. Lindemann, P.I. Bird,
R.W. Johnstone, J.A. Trapani, A central role for Bid in granzyme Binduced apoptosis, J. Biol. Chem. 280 (2005) 4476-4482.

[51] R.V. Talanian, X.H. Yang, J. Turbow, P. Seth, T. Ghayur, C.A. Casiano, K. Orth, C.J. Froelich, Granule-mediated killing: pathways for granzyme B-initiated apoptosis, J. Exp. Med. 186 (1997) 1323-1331.

[52] K.A. Sedelies, A. Ciccone, C.J. Clarke, J. Oliaro, V.R. Sutton, F.L. Scott, J. Silke, O. Susanto, D.R. Green, R.W. Johnstone, P.I. Bird, J.A. Trapani, N.J. Waterhouse, Blocking granule-mediated death by primary human NK cells requires both protection of mitochondria and inhibition of caspase activity, Cell Death Differ. 15 (2008) 708-717.

[53] I.S. Goping, T. Sawchuk, A. Rieger, I. Shostak, R.C. Bleackley, Cytotoxic T lymphocytes overcome Bcl-2 inhibition: target cells contribute to their own demise, Blood 111 (2008) 2142-2151.

[54] J.A. Heibein, M. Barry, B. Motyka, R.C. Bleackley, Granzyme B-induced loss of mitochondrial inner membrane potential (Delta Psi m) and cytochrome c release are caspase independent, J. Immunol. 163 (1999) 4683-4693.

[55] A. Mullbacher, R. Wallich, R.W. Moyer, M.M. Simon, Poxvirus-encoded serpins do not prevent cytolytic $\mathrm{T}$ cell-mediated recovery from primary infections, J. Immunol. 162 (1999) 7315-7321.

[56] H. Johnson, L. Scorrano, S.J. Korsmeyer, T.J. Ley, Cell death induced by granzyme C, Blood 101 (2003) 3093-3101.

[57] K. Ebnet, J. Chluba-de Tapia, U. Hurtenbach, M.D. Kramer, M.M. Simon, In vivo primed mouse $\mathrm{T}$ cells selectively express $\mathrm{T}$ cellspecific serine proteinase- 1 and the proteinase-like molecules granzyme B and C, Int. Immunol. 3 (1991) 9-19.

[58] E. Fellows, S. Gil-Parrado, D.E. Jenne, F.C. Kurschus, Natural killer cellderived human granzyme $\mathrm{H}$ induces an alternative, caspase-independent cell death program, Blood (2007).

[59] Q. Hou, T. Zhao, H. Zhang, H. Lu, Q. Zhang, L. Sun, Z. Fan, Granzyme $\mathrm{H}$ induces apoptosis of target tumor cells characterized by DNA fragmentation and Bid-dependent mitochondrial damage, Mol. Immunol. 45 (2008) 1044-1055.

[60] S. Mahrus, C.S. Craik, Selective chemical functional probes of granzymes A and B reveal granzyme B is a major effector of natural killer cell-mediated lysis of target cells, Chem. Biol. 12 (2005) 567-577.

[61] D.R. Hostetter, C.R. Loeb, F. Chu, C.S. Craik, Hip is a pro-survival substrate of granzyme B, J. Biol. Chem. 282 (2007) 27865-27874.

[62] T. Zhao, H. Zhang, Y. Guo, Z. Fan, Granzyme K directly processes Bid to release cytochrome $\mathrm{c}$ and endonuclease $\mathrm{G}$ leading to mitochondriadependent cell death, J. Biol. Chem. 282 (2007) 12104-12111.

[63] T. Zhao, H. Zhang, Y. Guo, Q. Zhang, G. Hua, H. Lu, Q. Hou, H. Liu, Z. Fan, Granzyme K cleaves the nucleosome assembly protein SET to induce single-stranded DNA nicks of target cells, Cell Death Differ. (2006).

[64] I.S. Goping, M. Barry, P. Liston, T. Sawchuk, G. Constantinescu, K.M. Michalak, I. Shostak, D.L. Roberts, A.M. Hunter, R. Korneluk, R.C. Bleackley, Granzyme B-induced apoptosis requires both direct caspase activation and relief of caspase inhibition, Immunity 18 (2003) $355-365$.

[65] V.R. Sutton, M.E. Wowk, M. Cancilla, J.A. Trapani, Caspase activation by granzyme B is indirect, and caspase autoprocessing requires the release of proapoptotic mitochondrial factors, Immunity 18 (2003) 319-329.

[66] M.R. Jenkins, K. Kedzierska, P.C. Doherty, S.J. Turner, Heterogeneity of effector phenotype for acute phase and memory influenza A virusspecific CTL, J. Immunol. 179 (2007) 64-70.

[67] M.R. Jenkins, J.A. Trapani, P.C. Doherty, S.J. Turner, Granzyme K expressing cytotoxic $\mathrm{T}$ lymphocytes protects against influenza virus in granzyme AB-/- mice, Viral Immunol. 21 (2008) 341-346.

[68] G. Hua, Q. Zhang, Z. Fan, Heat shock protein 75 (TRAP1) antagonizes reactive oxygen species generation and protects cells from granzyme Mmediated apoptosis, J. Biol. Chem. (2007).

[69] H. Lu, Q. Hou, T. Zhao, H. Zhang, Q. Zhang, L. Wu, Z. Fan, Granzyme M directly cleaves inhibitor of caspase-activated DNase (CAD) to unleash CAD leading to DNA fragmentation, J. Immunol. 177 (2006) 1171-1178.

[70] J.M. Kelly, N.J. Waterhouse, E. Cretney, K.A. Browne, S. Ellis, J.A. Trapani, M.J. Smyth, Granzyme M mediates a novel form of perforin-dependent cell death, J. Biol. Chem. (2004). 
[71] S.P. Cullen, I.S. Afonina, R. Donadini, A.U. Luthi, J.P. Medema, P.I. Bird, S.J. Martin, Nucleophosmin is cleaved and inactivated by the cytotoxic granule protease granzyme M during NK cell-mediated killing, J. Biol. Chem. (2008).

[72] N. Bovenschen, P.J. de Koning, R. Quadir, R. Broekhuizen, J.M. Damen, C.J. Froelich, M. Slijper, J.A. Kummer, NK cell protease granzyme M targets alpha-tubulin and disorganizes the microtubule network, J. Immunol. 180 (2008) 8184-8191.

[73] L.I. Pao, N. Sumaria, J.M. Kelly, S. van Dommelen, E. Cretney, M.E. Wallace, D.A. Anthony, A.P. Uldrich, D.I. Godfrey, J.M. Papadimitriou, A. Mullbacher, M.A. Degli-Esposti, M.J. Smyth, Functional analysis of granzyme M and its role in immunity to infection, J. Immunol. 175 (2005) 3235-3243.

[74] L.E. Sower, C.J. Froelich, N. Allegretto, P.M. Rose, W.D. Hanna, G.R. Klimpel, Extracellular activities of human granzyme A. Monocyte activation by granzyme A versus alpha-thrombin, J. Immunol. 156 (1996) $2585-2590$.

[75] L.E. Sower, G.R. Klimpel, W. Hanna, C.J. Froelich, Extracellular activities of human granzymes. I. Granzyme A induces IL6 and IL8 production in fibroblast and epithelial cell lines, Cell. Immunol. 171 (1996) 159-163.

[76] M.M. Simon, M.D. Kramer, Granzyme A, Meth. Enzymol. 244 (1994) 68-79.

[77] J.E. Knickelbein, K.M. Khanna, M.B. Yee, C.J. Baty, P.R. Kinchington, R.L. Hendricks, Noncytotoxic lytic granule-mediated CD8 $+\mathrm{T}$ cell inhibition of HSV-1 reactivation from neuronal latency, Science 322 (2008) 268-271.
[78] V. Romero, F. Andrade, Non-apoptotic functions of granzymes, Tissue Antigens 71 (2008) 409-416.

[79] J.C. Choy, V.H. Hung, A.L. Hunter, P.K. Cheung, B. Motyka, I.S. Goping, T. Sawchuk, R.C. Bleackley, T.J. Podor, B.M. McManus, D.J. Granville, Granzyme B induces smooth muscle cell apoptosis in the absence of perforin: involvement of extracellular matrix degradation, Arterioscler. Thromb. Vasc. Biol. 24 (2004) 2245-2250.

[80] J.C. Choy, R.P. Cruz, A. Kerjner, J. Geisbrecht, T. Sawchuk, S.A. Fraser, D. Hudig, R.C. Bleackley, F.R. Jirik, B.M. McManus, D.J. Granville, Granzyme B induces endothelial cell apoptosis and contributes to the development of transplant vascular disease, Am. J. Transplant. 5 (2005) 494-499.

[81] M.S. Buzza, L. Zamurs, J. Sun, C.H. Bird, A.I. Smith, J.A. Trapani, C.J. Froelich, E.C. Nice, P.I. Bird, Extracellular matrix remodeling by human granzyme B via cleavage of vitronectin, fibronectin, and laminin, J. Biol. Chem. 280 (2005) 23549-23558.

[82] J. Pardo, R. Wallich, K. Ebnet, S. Iden, H. Zentgraf, P. Martin, A. Ekiciler, A. Prins, A. Mullbacher, M. Huber, M.M. Simon, Granzyme $B$ is expressed in mouse mast cells in vivo and in vitro and causes delayed cell death independent of perforin, Cell Death Differ. 14 (2007) 1768-1779.

[83] C.J. Froelich, J. Pardo, M.M. Simon, Granule-assocated serine proteases: gzms might just not be killer proteases, J. Immunol. 30 (2009) 117-123. 